a loop of bowel, which has become fixed at its base by adhesions, becoming rotated, and so forming a volvulus. Obstruction may be caused, too, by obliteration of the lumen of the bowel, by contracting "bands" of fibrous tissue, or by strangulation of a loop of gut. That these dangers are not purely imaginary is shown in a paper by Dr. C. A. McWilliams ${ }^{1}$ who has been able to collect and analyse reports of 86 cases of intestinal obstruction resulting from appendicitis. From a study of these he concludes that obstruction is most likely to follow when there has been abscess formation; this was so in 81 per cent. of his cases. In 10 per cent. no operation had been performed, and in 9 per cent. there had been an "interval" operation-i.e., removal of the appendix after subsidence of an acute inflammatory attack. The onset of obstruction varied from within a few days of the appendix operation to seven years after. In all the cases where the seat of obstruction was mentioned it was in the small intestine. Dr. McWilliams' observations form one more indication for early operation in appendicitis, and in order that the chances of subsequent intestinal obstruction may be minimised, he advises that there should be as little handling of the intestines as possible, and that, whenever feasible, drainage should be avoided.

$$
1 \text { Medical News, Sspt } 3 .
$$

\section{FRACTURES AND DISLOCATIONS OF}

\section{THE CARPAL BONES.}

As a consequence of the increasing use of skiagraphy for diagnostic purposes, it is becoming daily more apparent that many injuries which have formerly been regarded as instances of contused bone, or have been included under the vague term "sprain," are in reality cases of fracture. Thus numerous examples could be quoted of complete transverse fractures of the long bones, where the usual and manifest signs of fracture have been absent, owing, presumably, to the integrity of the periosteum ; and many of the so called "sprains" about the hip, ankle, and wrist are now found to be associated with broken bones. It is important to urge this fact upon the profession; indeed, it may be enunciated as one of the guiding principles in medical practice to submit every case of "sprain" or "contused bone" to a competent skiagrapher whenever such a course is possible, both as a useful help towards diagnosis, and as a measure of protection to the medical attendant. The grave injury that may be inflicted on the doctor, especially in country districts and small towns, by an erstwhile patient whom he has treated for a "sprain," and who, because of prolonged pain and disability of the joint, subsequently discovers through an independent $x$-ray examination that a fracture is present, can scarcely be exaggerated.

After these preliminary remarks we may usefully draw attention to a paper by Dr. W. S. Haughton have observed. P. Holt, ${ }^{1}$ R.A.M.C. These writers have observed a number of cases of injuries to the wrist where the $x$ rays have revealed fracture or disproximal row on more of the carpal bones of the proximal row ; and their experience has led them to put forward the proposition that, with a history of on attemury to the wrist followed by extreme pain deformity, or with a history of old injury followed by more or less complete and permanent impairment of function, there will be found in almost all cases either fracture or more or less complete dislocation of one or more of the carpal bones

The violence necessary to produce these injuries is not necessarily extreme, and it varies greatly in form. Among the 10 cases which they record there was fracture of the scaphoid bone in eight, and there are some features connected with this injury that seem to be fairly characteristic. The injured hand is held in a position of extension and adduction, with marked resistance to attempts to abduct or flex the hand, while the point of greatest tenderness is over the scaphoid. The fracture is most commonly a transverse one through the middle of the bone, where the circumference is smallest.

Treatment, in cases of simple fracture without displacement, consists of massage and movement; and these should be repeated daily from the first. When a fragment is displaced it should be removed. When one or more bones are dislocated and cannot be reduced, excision through a dorsal incision is indicated, and a useful joint will result. With regard to the taking of the $x$ ray photographs, the authors lay stress on the value of stereoscopic skiagrams, and advise that in difficult cases, for purposes of comparison, the two wrists should be photographed in the following positions. (1) both prone, (2) both fully supinated, (3) both semiprone; and they add that it is absolutely necessary, for all comparative work, that some definite and constant points should be invariably made use of over which to place the anti.kathode.

$$
1 \text { Jour. R.A.M.C., September, } 1904 .
$$

\section{OSTEOPLASTIC AMPUTATIONS.}

IN the days before anæsthetics and antiseptics were used, a large part of the surgeon's operative experience was confined to amputations of the extremities, and this branch of work was perhaps brought as nearly to the height of perfection as was possible with the limitations imposed by the necessity for rapidity of procedure and the certain expectation of ensuing sepsis. Now, however, the surgeon is not only able to carry out carefully-planned and prolonged operations, but in most cases he may rely upon aseptic healing of the wound. With these two new principles to help, it would not have been surprising to observe a corresponding alteration of technique; and to some extent this has occurred; for example, transfixion methods and thick muscular flaps have given place to skin flaps with circular division of muscle. So far some improvement must. be admitted; but it is none the less true that there still remains much room for advancement in the technique of amputations, a fact to which the large proportion of tender and painful stumps seen in out-patient rooms and elsewhere bears eloquent. testimony. No doubt the superior fascinations of abdominal surgery and the great reduction of cases in which amputation is now necessary, thanks to the assistance rendered to conservative treatment by antiseptic methods, may be adduced to explain the anomaly. Explanations and excuses, however, can be placed on one side, and while urging that a more critical study should be given to the general subject of amputations, and more care should be devoted to following up such cases after they leave 